\section{Schizophrenia and comorbid substance abuse substantially increases risks of violent crime}

\section{QUESTION}

Question: What is the risk of violent crime in people with schizophrenia, and does substance abuse mediate this risk?

People: 8003 people (5243 men, 2760 women, 4674 with unaffected full siblings) aged 15 years or older with discharges from two or more hospitalisations for schizophrenia (ICD-8, ICD-9 and ICD-10 criteria) between 1 January 1973 and 31 December 2006. Diagnoses of schizophrenia were identified from the Hospital Discharge Register (HDR). Ten age and gender matched controls were selected from the general population for each person with schizophrenia (80 025 controls). 8123 full sibling controls were compared with the 4674 people with schizophrenia with unaffected full siblings.

Setting: Population study, Sweden; recruitment January 1973-December 2006.

Risk factors: Data on admissions for drug abuse or dependence and alcohol abuse or dependence (ICD-8, ICD-9 and ICD-10 criteria) were collected from the HDR. Data on convictions for violent crime (see outcomes for definition) were collected from the National Crime Register for people with schizophrenia and controls. Data on people with schizophrenia were merged with the Multi-Generation Register to find information about their siblings. The siblings were then linked to the HDR to find information on alcohol use disorders and the National Crime Register for information on convictions for violent offences. Data on income, marital status and immigration status were collected from the 1970 and 1990 national censuses.

Outcomes: Convictions for violent crime which included attempted homicide, common assault, aggravated assault (life threatening or causing severe bodily harm), robbery, arson, illegal threats, intimidation, sexual offense (rape, sexual coercion, child molestation) and sexual harassment including indecent exposure.

\section{METHODS \\ Design: Prospective cohort study. \\ Follow-up period: Up to 33 years.}

\section{MAIN RESULTS}

$1054(13.2 \%)$ of people with schizophrenia were convicted for a violent crime during follow-up compared with 4276 $(5.3 \%)$ of the general population controls (adjusted odds ratio (OR) 2.0, 95\% CI 1.8 to 2.2). 1959 people with schizophrenia had comorbid substance abuse $(25.5 \%)$ compared with $1863(2.3 \%)$ of the general population controls. Risk of violent crime increased in people with schizophrenia and comorbid substance abuse compared with people with schizophrenia alone $(27.6 \%$ vs $8.3 \%$, adjusted OR 4.4, 95\% CI 3.90 to 5.0 , see webextra table). Risk of violent crime in people with schizophrenia but without comorbid substance abuse compared with the general population controls $(8.5 \%$ vs $5.3 \%$, adjusted OR 1.2, 95\% CI 1.1 to 1.4). The risk increase for violence among people with schizophrenia and substance abuse comorbidity was reduced when unaffected siblings were used as controls $(28.3 \%$ vs $17.9 \%$; OR $1.8,95 \%$ I 1.4 to 2.4 , $\mathrm{p}<0.001$ ) (see webextra table).

\section{CONCLUSIONS}

Schizophrenia is associated with an increased risk of violent crime. Risk of violent crime is substantially increased among people with schizophrenia and comorbid substance abuse but only slightly increased in people with schizophrenia without comorbid substance abuse.

\section{ABSTRACTED FROM}

Fazel S, Langstrom N, Hjern A, et al. Schizophrenia, substance abuse, and violent crime. JAMA 2009;301:2016-23.

Correspondence to: Seena B Fazel, MD, University Department of Psychiatry, Warneford Hospital, 0xford, UK; seena.fazel@psych.ox.ac.uk

Sources of funding: Swedish Research Council-Medicine, Swedish Council for Working Life and Social Research.

- An additional table is published online only at http://ebmh.bmj.com/content/ vol13/issue 1
Patients with schizophrenia are often stigmatised which is a serious problem affecting the lives of many. One of the most important reasons for this is their perceived danger to society. Even mental health professionals sometimes overemphasise the threat. This paper showed where the bigger danger lays substance abuse. In addition to destigmatisation, this paper is also significant because of its clear message to treatment providers: treat substance abuse! The uncomfortable truth of the matter is that you have to treat the whole person-schizophrenia, substance abuse and all.

The association between schizophrenia and violence has been long debated but during the past years it has been considered a given. This register based study proved that the association is minimal in the absence of substance abuse comorbidity. It is not as simple a matter as some would like to think. Also, the novel addition of unaffected siblings showed that there are other underlying reasons for violence - the 'familial confounding'. One can only hope that this study gains enough attention to affect public and professional notions.

The authors are cautionary about their finding of those whose substance abuse was diagnosed after schizophrenia being more violent than those whose comorbidity was diagnosed before or simultaneously with schizophrenia. No matter how this result arose, it does point to the treatment services. We must do a better job treating people with several problems. Treating patients with schizophrenia and doing it skilfully has long lasting and widespread effects. There are no reasons for not doing it.
The study methodology is ingenious. When you have such reliable and all encompassing registers, this is exactly how they need to be used; simple, yet multifaceted, and with invaluable clinical implications. The Nordic countries are, of course, unique in not only their registers allowing such methodology but also in general societal issues. Yet, in countries with higher crime rates than Sweden, the relative impact of schizophrenia can be considered even less. Truth be told, Finns have a 'younger brother complex' in relation to the Swedes and I am convinced that there are now even more Finnish researchers saying: "Why didn't we do this?!"

Hanna Putkonen, MD, PhD

Vanha Vaasa hospital, Vaasa, Finland

Competing interests: None 